\title{
Formas metodológicas de avaliação em idosos institucionalizados: uma revisão sistemática
}

\author{
Methodolgical evaluation options of institutionalized elderly: a review
}

\author{
Aline Aparecida Chociai de Lima, ${ }^{1}$ Andressa Camargo, ${ }^{1}$ Carina Garçoa Raulik, ${ }^{1}$ \\ Debora Boguchewski Campos, ${ }^{1}$ Wagner Menna Pereira, ${ }^{1,2}$ \\ 'Universidade Estadual do Centro-Oeste (UNICENTRO), Guarapuava, PR, Brasil. \\ 2Faculdade Guairacá (SESG), Guarapuava, PR, Brasil.
}

Recebido em: 23/09/2016 / Aceito em: 24/05/2017 / Publicado em: 30/06/2017 wagner.fisio@hotmail.com

\section{RESUMO}

O envelhecimento é um processo fisiológico, o qual pode deixar o indivíduo propenso a situações de dependência em atividades de vida diária. Objetivo: elaborar uma revisão sobre as diversas formas metodológicas de avaliação em idosos institucionalizados, para que esta possa ser um instrumento informativo complementar o qual favorecerá o conhecimento e poderá ser usado como suporte para os profissionais, tendo em vista a escassez de estudos realizados com tal intuito. Método: trata-se de uma revisão sistemática da literatura. Para sua realização foram utilizadas como ferramenta de busca as bases de dados LILACS, Scielo, Pubmed e Google Acadêmico. Foram selecionados artigos que utilizaram testes e questionário validados em sua metodologia. A busca foi realizada entre os idiomas Português e Inglês, utilizando os descritores "idoso", "Instituição de longa permanência para idosos", "fisioterapia", "questionário". Resultado: foram selecionados 11 artigos, entre os quais foram descritos um total de 23 formas de avaliação, sendo elas: Mini Exame do Estado Mental (MEEM), Índice de Barthel, Timed Up And Go, Escala Funcional de Berg, Fallrisk score, Teste de Fluência Verbal Semântica, Bateria de Avaliação Frontal, Teste do Desenho do relógio, Escala da Atividade de Vida diária de Katz, Índice de Pfeffer, APGAR da família, Escala de Depressão Geriátrica, Inventário de Ansiedade Traço-Estado (IDATE), Teste de Tempo de Reação (TTR), Escala de Eficácia de Quedas (EEQ), Questionário WhoqolBref, Teste Físico AAHBRD adaptado para idosos institucionalizados, Teste da TriIha A, Escala da Demência (CDR), Escala de Fragilidade de Edmonton, International Consulationon Incontinency Questionnaire-Short Form, Questionário de Baecke e Teste de Caminhada de 6 minutos. Considerações finais: conclui-se que as formas de avaliação aqui apresentadas são de suma importância para conhecimento das afecções que podem acometer idosos institucionalizados, bem como, para direcionamento de tratamento. Palavras-chave: Idoso; Instituição de Longa Permanência para Idosos; Fisioterapia; Questionário.

\section{ABSTRACT}

Aging is a physiological process which can leave the individual prone to situations of dependence in activities of daily living. Objective: to elaborate a review on the different methodological forms of evaluation in institutionalized elderly, so that it can be a complementary information instrument which will favor the knowledge and can be used as a support for professionals, due to the shortage of studies carried out for this purpose. Method: this is a literature review. LILACS, Scielo, Pubmed and Google Academic databases were used as searching tools. We selected articles that use validated tests and questionnaires in their methodology. The search was performed between the Portuguese and English languages, using the descriptors "elderly", "Homes for the aged", "physiotherapy", and "questionnaire". Results: 11 articles were selected, among which a total of 23 evaluation forms were described, which is: Mini Mental State Examination (MMSE) of Folstein, Barthel Index, Timed Up And Go, Functional Berg Scale, Fallrisk score, Verbal Fluency semantic Test, Frontal Assessment Battery, clock Drawing Test, Scale of daily Life Activity Katz, Pfeffer Index, family APGAR, Depression Scale geriatric, Trait Anxiety Inventory-State (STAI), Reaction Time Test (RTT), Falls Efficacy Scale (FES), Whoqol- 
Bref Questionnaire, Physical Test AAHBRD adapted for institutionalized elderly, the track the Test Scale Demencia $(C D R)$, Fragility Scale Edmonton International Consulationon incontinency Questionnaire-Short Form Questionnaire Baecke and 6-minute walk test. Closing remarks: the conclusion is that the options for evaluation presented here are of fundamental importance for knowledge of the affections that can commit institutionalized elderly as well as for treatment orientation.

Keywords: Elderly; Homes for the Aged; Physiotherapy; Questionnaire.

\section{INTRODUÇÃO}

O envelhecimento pode ser definido como o conjunto de modificações que ocorrem num organismo ao longo do tempo, das quais podem ser citadas as alterações teciduais, celulares e em órgãos, que acarretam em uma maior probabilidade de morte. ${ }^{1}$ É um processo delicado para muitas pessoas, o que pode acarretar em isolamento social e agravamento de doenças, isso leva o idoso a precisar de cuidados. As instituições de longa permanência (ILPI) tornam-se a alternativa para esses indivíduos, oferendo uma condição de vida mais favorável a este idoso. ${ }^{2}$

O fenômeno conhecido por envelhecimento populacional está ocorrendo em todo o mundo. Em 2015 já existiam 901 milhões de pessoas com 60 anos ou mais, o que que representa $12 \%$ da população mundial. Estima-se que a população idosa está crescendo a uma taxa de 3,26 por cento ao ano. ${ }^{3}$

Ao saber sobre o considerável aumento que a população idosa vem tendo nos últimos anos, as preocupações acerca da qualidade de vida, dessa população, também têm aumentado entre os profissionais de saúde, pois as diversas alterações que ocorrem em consequência do envelhecimento levam a uma maior prevalência de fatores de risco e a ocorrência de doenças crônico-degenerativas. ${ }^{4}$ A soma de todas as alterações pode levar o idoso a ter uma redução da capacidade funcional, maior risco de quedas, alterações no equilíbrio, no tempo de reação e no sono; desta forma, podendo levar a um aumento da morbidade e mortalidade nessa população. ${ }^{5}$ A avaliação da capacidade funcional e da qualidade de vida dos idosos se faz fundamental na determinação do perfil dessa população, na criação de programas de intervenção e na definição de níveis de morbidade e mortalidade dos mesmos. ${ }^{6}$

Tomando conhecimento sobre o aumento do número de idosos nos últimos anos, bem como, da importância da realização de uma avaliação funcional nessa população, levando em consideração a escassez de trabalhos relacionados ao referente assunto na literatura, o presente estudo teve como objetivo reunir as diferentes formas metodológicas encontradas na literatura de avaliação em idosos, visando um maior conhecimento sobre as mesmas, para que assim possam ser utilizadas como estratégias e diretrizes no planejamento de condutas e planos de tratamento adequados para essa população.

\section{MÉTODO}

O presente estudo trata-se de uma revisão sistemática da literatura sobre as diferentes formas de avaliação em idosos institucionalizados. Para realização deste trabalho foram selecionados artigos que utilizaram testes e questionários validados em sua metodologia.

A busca conduziu-se através das bases de dados LILACS, Scielo, Pubmed, Google Acadêmico, no período de 1 de Agosto a 12 Setembro de 2016. A busca foi realizada entre os idiomas Português e Inglês, utilizando os descritores "idoso", "Instituição de longa permanência para idosos", "fisioterapia", "questionário".

Foram utilizados, como critério de exclusão, os estudos cuja data excedia 5 anos, a partir do início desta pesquisa, artigos que comparavam idosos institucionalizados e não institucionalizados, e também aqueles que não possuíam, uma forma de avaliação validada. Como critério de inclusão utilizaram-se artigos que possuíam em sua metodologia, a avaliação do estado cognitivo, psicológico, funcional, equilíbrio, força, qualidade de vida, qualidade do sono e marcha.

Para seleção da amostra inicial, a busca partiu de uma leitura minuciosa de títulos e resumos e, caso este não fosse esclarecedor uma leitura na integra fez-se necessária. Através da pesquisa, obteve-se um total de 27 artigos e, considerando os parâmetros de seleção já mencionados, observou-se que 11 artigos seguiram os critérios estipulados, trabalhos estes que utilizaram as seguintes formas de avaliação em idosos: Mini Exame do Estado Mental (MEEM) de Folstein, Indice de Barthel, Timed Up And Go, Escala Funcional de Berg, Fallrisk score, Teste de Fluência Verbal Semântica, Bateria de Avaliação Frontal, Teste do Desenho do relógio, Escala da Atividade de Vida diária de Katz, Índice de Pfeffer, APGAR da família, Escala de Depressão Geriátrica, Inventário de Ansiedade Traço-Estado (IDATE), Teste de Tempo de Reação (TTR), Escala de Eficácia de Quedas (EEQ), Questionário WhoqolBref, Teste Físico AAHBRD adaptado para idosos institucionalizados, Teste da Trilha A, Escala da Demência (CDR), Escala de Fragilidade de Edmonton, International Consulationon Incontinency Questionnaire-Short Form, Questionário de Baecke e Teste de Caminhada de 6 minutos.

\section{RESULTADOS}

Para a realização desta revisão sistemática, foram selecionados e analisados 11 estudos, os quais apresentavam diversas formas de avaliação realizadas em idosos institucionalizados. Todos os estudos foram publicados em revistas indexadas. Os periódicos com publicação foram: Dove Press, Revista inspirar, Revista fisioterapia em movimento, Acta paulista de Enfermagem, Revista estudos interdisciplinares sobre o envelhecimento, Revista brasileira de cineantropometria e desempenho humano, Escola Anna Nery Revista de Enfermagem, Revista Baiana de Enfermagem, Revista Brasileira de Atividade Física e saúde. Revista da Escola de Enfermagem e Revista Brasileira de enfermagem.

Na tabela 1 estão apresentados os artigos sobre as formas metodológicas de avaliação em idosos institucionalizados encontradas, abordando os autores responsáveis pela publicação, título do trabalho, ano da publicação, desenho metodológico aplicado e métodos de avaliação utilizados. Os artigos encontrados, foram publicados entre os anos de 2011 e 2015.

No total, 937 idosos institucionalizados foram incluídos nos 11 estudos e foram identificados um total de 23 métodos de avaliação. 
Tabela 1 - Estudos analisados metodologicamente para comparação sistemática.

\begin{tabular}{|c|c|c|c|c|c|}
\hline AUTORES & TÍTULO & ANO & METODOLOGIA & MÉTODO DE AVALIAC̣ÃO & CONCLUSÃO \\
\hline $\begin{array}{l}\text { Luciana de Araújo } \\
\text { Reis }{ }^{1}, \text { Thaís de } \\
\text { Souza Rocha }{ }^{2} \text { e } \\
\text { Stênio Fernando } \\
\text { Pimentel Duarte }^{3}\end{array}$ & $\begin{array}{l}\text { Quedas: Risco e } \\
\text { fatores associados } \\
\text { em idosos institu- } \\
\text { cionalizados }\end{array}$ & 2014 & $\begin{array}{l}\text { A amostra do estudo foi } \\
\text { constituída por todos os } \\
\text { idosos residentes nas ILPs que } \\
\text { tiveram condições mentais para } \\
\text { responder ao instrumento de } \\
\text { pesquisa, perfazendo um total } \\
\text { de } 62 \text { idosos. }\end{array}$ & $\begin{array}{l}\text { Mini Exame do Estado Mental } \\
\text { (MEEM) de Folstein, índice de } \\
\text { Barthel, Timed Up and Go, } \\
\text { Escala Funcional de Berg e } \\
\text { Fall Risk Score }\end{array}$ & $\begin{array}{l}\text { Verificou-se diferença estatís- } \\
\text { tica significativa entre as var- } \\
\text { iáveis presença de problemas } \\
\text { de saúde, presença de sequelas } \\
\text { e comprometimento das ativi- } \\
\text { dades básicas de vida diária na } \\
\text { Escala de Berg }\end{array}$ \\
\hline
\end{tabular}

Aghlen de Souza Efeitos de uma Martins ${ }^{1}$, Rafael terapia cognitivoCosta Freire ${ }^{1}$, Luiza motora em idosos Helena Martins ${ }^{1}$, institucionalizados Brunna Berton', Silvio Assis Oliveira Júnior ${ }^{2}$ e Gustavo Christofoletti ${ }^{3}$
2014 Pesquisa empírica quantitativa do tipo ensaio clínico com dois grupos independentes: grupo experimental (GE) e grupo controle (GC). Os participantes foram selecionados em ILPI da cidade de Campo Grande/MS.
Mini Exame do Estado Mental, Teste de Fluência Verbal Semântica, Bateria de Avaliação Frontal e Teste do Desenho do Relógio; Escala de Equilíbrio Funcional de Berg, Timed Up and Go, Escala de Atividades da Vida Diária de Katz e Índice de Pfeffer.

\section{Liliane Dias e Dias de Macedo ${ }^{1}$, Thaís Cristina Galdino de Oliveira ${ }^{1}$, Fernanda Cabral Soares ${ }^{1}$ João Bento-Torres ${ }^{1}$, Natáli Valim Oliver Bento-Torres ${ }^{1}$, Dan- iel Clive Anthony ${ }^{3}$, Cristovam Wander- ley Picanço-Diniz ${ }^{1}$}

Simone Camargo

\section{Simone Camargo} de Oliveira ${ }^{1}$, Ariene Angelini dos Santos $^{2}$ e Sofia Cristina lost Pavarini ${ }^{3}$

\section{Beneficial effects} of multisensory and cognitive stimulation in institutionalized elderly: 12-months follow-up

\section{Os participantes foram indi-} víduos com idade de 65 anos ou mais de idade, sem história de traumatismo craniano, acidente vascular cerebral, depressão ou alcoolismo crónico.
Relação entre sintomas depressivos e a funcionalidade familiar de idosos institucionalizados
2013 Foi realizado com idosos residentes em seis ILPIs de São Carlos - SP, sendo três privadas e três filantrópicas.
APGAR de família e a Escala de Depressão Geriátrica GDS
Mini Exame do Estado Mental.
O protocolo assistencial não foi eficaz sobre a função cognitiva dos participantes

A magnitude e duração dos benefícios adquiridos de paradigmas de estimulação para idosos são significativamente diferentes em idosos institucionalizados e não institucionalizados

Daianne Goncalves ${ }^{1}$, Caroline Altermann², Aline Vieira $^{3}$, Ana Paula Machado ${ }^{4}$, Rachel Fernandes ${ }^{5}$, Andressa Oliveira ${ }^{6} \mathrm{e}$ Pámela Billig MelloCarpes $^{7}$
Avaliação das fun- ções cognitivas, qualidade de sono, tempo de reação e risco de quedas em idosos institu- cionalizados
2013 A amostra utilizada foi com- posta por 10 idosos institu- cionalizados ( 7 mulheres e 3 homens), residentes em uma instituição particular voltada à classe de baixa renda do município de Uruguaiana-RS.
Mini exame do estado Mental (MEEM), Escala de Depressão Geriátrica (GDS), Inventário de Ansiedade Traço-Estado (IDATE), Teste de Tempo de Reação (TTR) e Escala de Eficácia de Quedas (EEQ).

A maioria dos idosos apresentou elevada disfunção familiar, seguida de boa funcionalidade familiar e moderada disfunção familiar. A presença de sintomas depressivos foi maior entre os idosos com disfunção familiar. Os resultados demonstraram também a existência de correlação significativa entre o APGAR de Família e os sintomas depressivos

\begin{tabular}{|c|c|c|c|c|c|}
\hline $\begin{array}{l}\text { Elizabete Re- } \\
\text { gina Araújo de Ol- } \\
\text { iveira'; Maria José } \\
\text { Gomes }^{2} \text {; Karina } \\
\text { Mary de Paiva }^{3}\end{array}$ & $\begin{array}{l}\text { Institucionalização } \\
\text { e qualidade de vida } \\
\text { de idosos da região } \\
\text { metropolitana de } \\
\text { Vitória - ES }\end{array}$ & 2011 & $\begin{array}{l}\text { Foram pesquisadas } 14 \text { insti- } \\
\text { tuições de longa permanên- } \\
\text { cia, sendo } 3 \text { no município de } \\
\text { Cariacica, } 4 \text { na Serra, } 5 \text { em } \\
\text { Vila Velha e } 2 \text { em Vitória. }\end{array}$ & Questionário Whoqolbref & $\begin{array}{l}\text { A qualidade de vida nos idosos } \\
\text { institucionalizados deste estudo } \\
\text { foi pior quando comparada } \\
\text { àqueles não institucionalizados } \\
\text { em todos os domínios avaliados }\end{array}$ \\
\hline $\begin{array}{l}\text { Tânia R. Bertoldo } \\
\text { Benedetti }{ }^{1} \text {, Giovana } \\
\text { Zarpellon Mazo², } \\
\text { Lucia Hisako Takase } \\
\text { Gonçalves }^{3}\end{array}$ & $\begin{array}{l}\text { Bateria de testes } \\
\text { da AAHPERD: } \\
\text { adaptação para } \\
\text { idosos institucion- } \\
\text { alizados }\end{array}$ & 2014 & $\begin{array}{l}\text { Amostra de } 92 \text { idosos } \\
\text { institucionalizados de cinco } \\
\text { ILPls localizados em distintos } \\
\text { contextos geográfico-cul- } \\
\text { turais: Florianópolis-SC, Porto } \\
\text { Alegre-RS, Rio Grande-RS, } \\
\text { Passo Fundo-RS, Ribeirão } \\
\text { Preto-SP e Jequié-BA. }\end{array}$ & $\begin{array}{l}\text { Testes físicos AAHPERD } \\
\text { adaptada para idosos } \\
\text { institucionalizados. }\end{array}$ & $\begin{array}{l}\text { A versão AAHPERD adaptada } \\
\text { e testada para idosos institu- } \\
\text { cionalizados demonstrou sua } \\
\text { aplicabilidade com possibili- } \\
\text { dade de avaliação da aptidão } \\
\text { funcional de idosos residentes } \\
\text { em ILPIs }\end{array}$ \\
\hline
\end{tabular}




\begin{tabular}{|c|c|c|c|c|c|}
\hline $\begin{array}{l}\text { Luzia Sousa Fer- } \\
\text { reira }^{1} \text {, Maria do } \\
\text { Socorro Pereira } \\
\text { Pinho }{ }^{1} \text {, Moisés } \\
\text { Wesley de Macedo } \\
\text { Pereira }^{2} \text {, Aparecido } \\
\text { Pimentel Ferreira }^{3}\end{array}$ & $\begin{array}{l}\text { Perfil cognitivo de } \\
\text { idosos residentes } \\
\text { em Instituições de } \\
\text { Longa Permanên- } \\
\text { cia de Brasília-DF }\end{array}$ & 2014 & $\begin{array}{l}\text { Sessenta idosos institucion- } \\
\text { alizados em Instituições de } \\
\text { Longa Permanência foram } \\
\text { submetidos à avaliações }\end{array}$ & $\begin{array}{l}\text { Mini Exame do Estado Mental } \\
\text { (MEEM), Teste de Trilha A e a } \\
\text { Escala de Demência (CDR). }\end{array}$ & $\begin{array}{l}\text { Os principais resultados do pre- } \\
\text { sente estudo demonstram que } \\
\text { houve perda cognitiva em um } \\
\text { grande número de idosos }\end{array}$ \\
\hline $\begin{array}{l}\text { Cíntia Lira Borges }^{1} \text {; } \\
\text { Maria Josefina da } \\
\text { Silva }^{2} \text {; Jorge Wilker } \\
\text { Bezerra Clares }^{1} ; \\
\text { Maria Eliana Pei- } \\
\text { xoto Bessa }{ }^{2} \text {; Maria } \\
\text { Célia de Freitas }^{1}\end{array}$ & $\begin{array}{l}\text { Avaliação da fra- } \\
\text { gilidade de idosos } \\
\text { institucionalizados }\end{array}$ & 2013 & $\begin{array}{l}\text { Realizado com } 54 \text { idosos } \\
\text { residentes em instituição de } \\
\text { longa permanência da região } \\
\text { Nordeste do Brasil. }\end{array}$ & $\begin{array}{l}\text { Escala de Fragilidade de } \\
\text { Edmonton. }\end{array}$ & $\begin{array}{l}\text { A fragilidade em idosos institu- } \\
\text { cionalizados sofre influência das } \\
\text { características sociodemográfi- } \\
\text { cas e clínicas. }\end{array}$ \\
\hline $\begin{array}{l}\text { Javier Jerez Roiga,b, } \\
\text { Dyego Leandro }^{a} \\
\text { Bezerra de Souza }^{a} \text {; } \\
\text { Kenio Costa Lima }\end{array}$ & $\begin{array}{l}\text { Urinary inconti- } \\
\text { nence in institu- } \\
\text { tionalized elderly: } \\
\text { prevalence and } \\
\text { impact on quality } \\
\text { of life }\end{array}$ & 2015 & $\begin{array}{l}\text { Foi desenvolvido em dez insti- } \\
\text { tuições de longa permanência } \\
\text { para idosos de Natal (Brasil). }\end{array}$ & $\begin{array}{l}\text { International Consultationon } \\
\text { Incontinence Questionnaire- } \\
\text { Short Form. }\end{array}$ & $\begin{array}{l}\text { A prevalência de incontinência } \\
\text { urinária nesta amostra com } \\
\text { capacidade cognitiva para } \\
\text { responder questionários foi de } \\
\text { mais de } 40 \% \text {, e a severidade } \\
\text { foi elevada. Aproximadamente } \\
\text { metade dos incontinentes } \\
\text { refere um impacto moderado ou } \\
\text { severo das perdas urinárias. }\end{array}$ \\
\hline $\begin{array}{l}\text { Flora Isabel Ra- } \\
\text { malho Leite Reis }{ }^{1} ; \\
\text { Marcelo Tavella } \\
\text { Navega }^{2,3} \text {; Robison } \\
\text { José Quitério } \\
\text { Alex; } \\
\text { Pexandre Ricardo } \\
\text { Pmbrozin²; }\end{array}$ & $\begin{array}{l}\text { Existe Correlação } \\
\text { Entre Força Mus- } \\
\text { cular com Teste } \\
\text { de Caminhada de } \\
\text { Seis Minutos em } \\
\text { Idosos Institucion- } \\
\text { alizados? }\end{array}$ & 2012 & $\begin{array}{l}\text { Foram incluídos nesta pes- } \\
\text { quisa } 9 \text { idosos institucionali- } \\
\text { zados de ambos os sexos. }\end{array}$ & $\begin{array}{l}\text { Mini Exame do Estado Mental } \\
\text { (MEEM), Questionário de } \\
\text { Baecke e TC6 }\end{array}$ & $\begin{array}{l}\text { Por um período de } 45 \text { dias de } \\
\text { imobilização as cartilagens } \\
\text { articulares do fêmur e da tíbia } \\
\text { sofrem alterações na população } \\
\text { de condrócitos e o aumento de } \\
\text { grupos isógenos nos fêmures } \\
\text { dos grupos experimentais tanto } \\
\text { em } 21 \text { como em } 45 \text { dias indi- } \\
\text { cam um pro- cesso de divisão } \\
\text { celular como mecanismo de } \\
\text { defesa adotado } \\
\text { pela cartilagem. }\end{array}$ \\
\hline
\end{tabular}

\section{DISCUSSÃO}

Reis, Rocha e Duarte ${ }^{7}$ realizaram uma pesquisa do tipo analítica com delineamento transversal e abordagem quantitativa com uma amostra constituída por todos 62 idosos residentes nas ILPIs que tiveram condições mentais para responder ao instrumento de pesquisa. Os autores analisaram o estado mental dos idosos, através do Mini Exame do Estado Mental (MEEM) de Folstein. O estudo foi composto por 5 etapas: Avaliação cognitiva: utilizou-se o MEEM de M. Folstein, Informações sociodemográficas, Estado de saúde, Capacidade funcional (CF) e Avaliação do risco de quedas através dos testes Timed Up and Go (TUG), Escala Funcional de Berg e Fall risk score de Downton.

O Mini-Exame do Estado Mental é um instrumento de avaliação de funções cognitivas composto por questões que avaliam orientação temporal e espacial, registro de palavras, atenção e cálculo, lembrança de palavras, linguagem e capacidade construtiva visual. 0 escore pode variar de zero até 30 pontos. Em indivíduos saudáveis são esperados escores superiores a 25; abaixo disso, há indício de perda da capacidade cognitiva, que pode ser leve (21-24 pontos), moderada (10-20) ou grave (escores inferiores ou iguais a 9). ${ }^{8}$

O Índice de Barthel é um instrumento utilizado para avaliar as atividades da vida diária (AVDs). O mesmo mede a independência funcional no cuidado pessoal, mobilidade, locomoção e eliminações. Em sua versão original, cada item é pontuado conforme o desempenho do paciente em realizar tarefas de forma independente, com alguma ajuda ou de forma dependente. Forma-se uma pontuação geral, através dos pontos de cada categoria, dependendo do tempo e da assistência necessária a cada paciente. A pontuação varia de 0 a 100 , em intervalos de cinco pontos, e as pontuações mais altas indicam maior independência. ${ }^{9}$

O teste Timed Up and Go (TUG), foi introduzido em 1991 por Podsiadlo e Richardson. ${ }^{10} \mathrm{O}$ procedimento descrito para a realização do teste documenta o tempo em segundos necessário para os indivíduos: Levantar a partir de uma cadeira de braço padrão, caminhar até uma linha no chão de 3 metros de distância, virar, volta, e sentar-se novamente. Na pontuação do teste, ao realizá-lo em menos de 10 segundos, sugere-se que os indivíduos são absolutamente livres e independentes, entre 10 e 19 segundos sugere que são independentes, por apresentar velocidade de marcha e equilíbrio razoável, e entre 20 e 29 segundos apresentam dificuldades para as atividades de vida diária. Numa pontuação de 30 ou mais segundos, os indivíduos tendem a ser totalmente dependentes para atividades da vida diária. ${ }^{11}$

A Avaliação do equilíbrio por meio da Escala de Equilíbrio de Berg (EEB), adaptada para a aplicação no Brasil, é um instrumento de avaliação amplamente utilizada em estudos envolvendo idosos, o qual contem 14 
itens que simulam atividades comuns de vida diária. Cada item possui 5 possibilidades de pontuação, variando de 0 a 4, totalizando o máximo de 56 pontos, com um ponto de corte de 45 pontos para risco de quedas. ${ }^{12}$ No estudo de Reis, Rocha e Duarte ${ }^{7}$, o teste Timed Up and Go, verificou que a menor parte dos idosos possui baixo risco de quedas; já, na escala de Berg, a maior parte dos idosos foi classificada com risco de quedas. Ambos os testes são bastante utilizados para avaliação do risco de quedas, porém a escala de Berg, além de ter aplicação mais demorada, exige maior treinamento do aplicador.

A Fall risk score de Downton, utiliza cinco critérios para fazer a avaliação do risco de quedas da população idosa: já sofreu quedas anteriormente; utiliza alguma medicação; apresenta algum déficit sensorial; avaliação do estado mental e avaliação da marcha. A pontuação do instrumento varia de 0 a 11, e o idoso é classificado com alto risco de queda quando apresenta uma pontuação igual ou superior a três. ${ }^{7}$ Devido os episódios de quedas serem um evento bastante frequente na população idosa, destaca-se a importância do conhecimento dos profissionais de saúde acerca dos mecanismos de queda, para que assim os fatores de risco possam ser identificados precocemente podendo assim haver implantações de estratégias de prevenção dos mesmos.

Martins et. al. ${ }^{13}$ realizaram uma pesquisa empírica quantitativa do tipo ensaio clínico com dois grupos independentes, grupo experimental (GE) e grupo controle (GC) a qual avaliava os efeitos de uma terapia cognitivo-motora em idosos institucionalizados. Foram selecionados 16 indivíduos com idade média de 76,56 $\pm 6,38$ anos. Para avaliação, foram aplicados os seguintes instrumentos: Mini-Exame do Estado Mental, Teste de Fluência Verbal Semântica, Bateria de Avaliação Frontal Teste do Desenho do Relógio para análise das funções cognitivas; Escala de Equilíbrio Funcional de Berg e Teste Timed Up and Go para verificação da mobilidade, e a Escala de Atividades da Vida Diária de Katz e Índice de Pfeffer para mensuração da independência funcional dos participantes.

A prova de fluência verbal fornece informações acerca da capacidade de armazenamento do sistema de memória semântica, da habilidade de recuperar a informação guardada na memória e do processamento das funções executivas, especialmente, aquelas através da capacidade de organizar o pensamento e as estratégias utilizadas para a busca de palavras. $O$ teste de fluência envolve a geração do maior número de palavras possíveis em período de tempo fixado. A fluência verbal semântica (FVS) exige maior ativação das regiões do lobo temporal e depende do acesso e da integridade da memória semântica, sendo esta um componente da memória de longo prazo que contém a representação permanente do nosso conhecimento sobre os objetos, fatos e conceitos, bem como palavras e seus significados. ${ }^{14}$ Os testes de FVS, são provas que solicitam que os sujeitos produzam e verbalizem o maior número de palavras possível, numa categoria semântica específica. As categorias semânticas mais utilizadas são: Animais, Alimentos, Frutos, Nomes Próprios de Rapazes e Moças, Peças de Vestuário, entre outros. ${ }^{15}$

A Bateria de Avaliação Frontal (BAF) foi desenvolvida com o objetivo de avaliar as funções cognitivas frontais, e proposta de modo recente como um breve diagnóstico em casos de disfunções executivas. Tem sido aplicada em pacientes com doença de Alzheimer, demência fronto-temporal e doença de Parkinson. É constituída por seis subtestes: raciocínio abstrato, flexibilidade mental, programação cognitiva para ação motora, sensibilidade à interferência, controle inibitório e autonomia no controle interno dos estímulos ambientais. Mensura em seus domínios: conceituação, flexibilidade mental, programação, sensibilidade e interferência, controle inibitório e autonomia ambiental. ${ }^{16}$

O Índice de Katz é baseado numa avaliação da independência ou dependência funcional dos pacientes para atividades como: tomar banho, se vestir, usar o sanitário, mover-se, ser continente e comer sem ajuda. O grau de dependência é classificado da seguinte forma: A: Independente para comer, ser continente, mover-se, usar o sanitário, se vestir e tomar banho; B: Independente para realizar todas as funções citadas, exceto uma; C: Independente para realizar todas as funções, exceto tomar banho e mais outra função; D: Independente para realizar todas as funções, exceto para tomar banho, se vestir e mais outra função; E: Independente para realizar todas as funções, exceto tomar banho, se vestir, usar o sanitário; F: Independente para realizar todas as funções, exceto tomar banho, se vestir, usar o sanitário, mover-se e mais outra função; G: Dependente para realizar todas as seis funções e; Outro: Dependente para realizar pelo menos duas funções, mas não pode ser classificado em C, D, E e F. ${ }^{17}$

Dias et. al. ${ }^{18}$ em seu estudo sobre os efeitos benéficos da estimulação multissensorial e cognitiva em idosos institucionalizados (grupo $\mathrm{I}, \mathrm{n}=20$ ), e não institucionalizados (grupo $\mathrm{NI}, \mathrm{n}=15$ ), utilizaram como forma de avaliação cognitiva o Mini Exame do Estado Mental. O tratamento foi composto por 48 sessões, realizadas duas vezes por semana, visando uma melhora no estado cognitivo. Ao término do tratamento, os indivíduos foram avaliados novamente e notou-se que o grupo de idosos não institucionalizados obteve uma resposta meIhor ao tratamento. Durante 1 ano, após a conclusão da intervenção, foram realizadas 5 reavaliações $(2$ meses, 4 meses, 6 meses, 8 meses, 12 meses). Foi observado um declínio cognitivo em ambos os grupos. Levou apenas de 4 a 6 meses após o fim do programa de estimulação para aparecerem reduções significantes na pontuação dos testes de linguagem. No entanto, reduções precoces na pontuação dos testes foram principalmente associadas ao grupo I. Tais achados mostram que idosos institucionalizados são mais suscetíveis a ter um pior estado cognitivo do que aqueles que vivem em comunidade.

A Escala de Depressão Geriátrica de Yesavage é composta por 30 itens com questões fechadas: "sim ou não". Sua pontuação total é de 30 pontos, e pontuações superiores ou iguais a 11 são indicativas de transtorno afetivo. $^{19}$

Rosseto et. al. ${ }^{20}$ avaliaram a presença de depressão em idosos residentes numa Instituição de Longa Permanência, onde através da aplicação da Escala de Depressão Geriátria (EDG-15), os resultados demonstraram que $25 \%$ dos idosos não apresentavam indícios de depressão; 43,75\% apresentavam indícios de depressão leve à moderada e $31,25 \%$ apresentavam indícios de depressão severa. Tais resultados foram capazes de 
mostrar a necessidade das ILPIs pensar em estratégias que objetivem alterar este quadro no que diz respeito ao apoio psicológico, proporcionando uma melhor qualidade de vida aos idosos.

Segundo Paiva et. al. ${ }^{21}$ o APGAR de família, é composto por cinco questões, uma para cada domínio de avaliação, cujas opções de respostas são: sempre (2), algumas vezes (1) e nunca (0), com a pontuação total variando de 0 a 10, com a classificação de 0 a 4, elevada disfunção familiar; de 5 a 6, moderada disfunção familiar; e de 7 a 10, boa funcionalidade familiar. Os idosos institucionalizados com sintomas depressivos eram predominantemente mulheres e estavam inseridos na faixa etária de 80 anos e mais. Com relação à funcionalidade familiar, a maioria dos idosos apresentou elevada disfunção familiar (57\%). A disfunção familiar foi maior entre os idosos com sintomas depressivos.

Gonçalves et. al. ${ }^{5}$ em seu estudo objetivaram avaliar o perfil cognitivo, o tempo de reação, o risco de quedas e a qualidade do sono de idosos institucionalizados da cidade de Uruguaiana-RS. Foram avaliados 10 idosos de ambos os sexos utilizando como instrumentos o Mini-Exame do Estado Mental (MEEM), a Escala de Depressão Geriátrica (EDG) e o Inventário de Ansiedade Traço-Estado. Inventário de Ansiedade Traço-Estado trata-se de um questionário de auto avaliação dividido em duas partes, a primeira avalia a ansiedade-traço e a segunda avalia a ansiedade-estado, sendo que cada parte contém 20 afirmações, o questionário possui uma escala de 4 itens que variam de 1 a 4 , sendo que ESTADO significa como o sujeito se sente no "momento" e o TRAÇO como ele "geralmente se sente". O escore de cada parte varia de 20 a 80 pontos, sendo que, quanto mais baixo o escore, menor é o grau de ansiedade). ${ }^{22}$

O Teste de Tempo de Reação (TTR) segundo Corazza, Pereira e $\operatorname{Dias}^{23}$ analisa o tempo de reação e do estado cognitivo em idosas praticantes de atividades físicas, por meio de um software com estímulo visual, utilizando somente os dois botões do mouse, dividido em dois subtipos, o TRS (Tempo de reação simples) realiza ao surgir na tela um círculo vermelho e em seguida um círculo verde, quando esse último aparece é o momento de responder ao sinal, portanto o sujeito que estará com um dos dedos da mão dominante (a escolher) segurando um dos botões do mouse, deverá soltá-lo. E o TRE (Tempo de reação de Escolha) possui a estrutura semelhante ao anterior, porém o sujeito estará diante do computador, tendo o "mouse", bem a sua frente. O sujeito estará posicionado com os dedos indicadores de ambas as mãos na tecla da direita e da esquerda, podendo surgir na tela a luz azul ou verde, para a primeira o indivíduo deverá responder acionando o dedo da mão direita e para a luz verde o dedo da mão esquerda).

A Escala de Eficácia de Quedas (EEQ), segundo a definição de Camargos et. al., ${ }^{24}$ trata-se de um questionário que contém 16 domínios com diferentes atividades de vida diária, com quatro possibilidades de respostas e respectivos escores de um a quatro ("Nem um pouco preocupado" a "Extremamente preocupado"). O escore total pode variar de 16 a 64 (ausência de preocupação a preocupação extrema) em relação às quedas, durante a realização das atividades específicas do questionário.

O Índice de Qualidade de Sono de Pittsburgh
(IQSP) segundo Chellapa e Araujo ${ }^{25}$ é composto por 19 itens, que são agrupados em sete componentes, cada qual pontuado em uma escala de 0 a 3 . Os componentes são, respectivamente: (1) a qualidade subjetiva do sono; (2) a latência do sono; (3) a duração do sono; (4) a eficiência habitual do sono; (5) as alterações do sono; (6) o uso de medicações para o sono; e (7) a disfunção diurna. Os escores dos sete componentes são somados para conferir uma pontuação global do PSQI, a qual varia de 0 a 21. Pontuações de 0-4 indicam boa qualidade do sono, de 5-10 indicam qualidade ruim e acima de 10 indicam distúrbio do sono.

A escala de Pfeffer, constituída por 10 itens, identifica a funcionalidade, através do grau de independência para realização das atividades instrumentais de vida diária. O escore mínimo é 0 e o máximo é 30 . Quanto maior a pontuação, maior é a dependência do paciente, considerando-se presença de prejuízo funcional, a partir de um escore de $3 .^{26}$

Gomes, Oliveira e Paiva ${ }^{27}$ realizaram uma pesquisa com 70 idosos que conseguiam realizar as atividades de vida diárias (AVD's) e que eram residentes em instituições que se em instituições de longa permanência e em domicílios particulares na região metropolitana de Vitória-ES com o objetivo de comparar a qualidade de vida desses indivíduos. Para comparação, um grupo de 210 idosos não institucionalizados foram adicionados ao estudo. Para a avaliação de AVD's utiliza-se o WHOQOL-100, que é composto por 24 particularidades, sendo agrupadas em quatro partes. Porém, WHOQOL-100, é um questionário longo e detalhado, então o WHOQOL-BREF acabou sendo mais proveitoso no estudo, por ser uma versão simplificada, no qual não tem a divisão das 4 partes, sendo substituído por apenas uma pergunta, as quais somadas com mais duas questões gerais da qualidade de vida, somam 26 questões. ${ }^{28}$

Benedetti et. al. ${ }^{29}$ realizaram a adaptação da bateria de testes AAHPERD, a partir de bases metodológicas com o objetivo de ser executado em idosos institucionalizados e definir valores que determinassem as habilidades funcionais para esses indivíduos. A bateria abrange cinco domínios físicos que são importantes para realização de tarefas no dia a dia. O teste de flexibilidade foi modificado para que o idoso não precisasse abaixar-se em um nível menor que de uma cadeira, por exemplo, pela dificuldade que alguns idosos apresentam em alguns movimentos e posicionamentos corporais. Uma tábua sustentada em duas cadeiras foi utilizada para adaptação da posição corporal. Então para realizar este teste o idoso sentava em uma cadeira e estendia as pernas sobre a tabua. $O$ teste de resistência aeróbia geral e habilidade para andar/caminhar foi trocado pela dificuldade de alguns idosos de cumprir a distância determinada pelo teste e também pela restrição de espaço apresentada na maioria das Instituição de longa permanência para idosos (ILPIs).

Ferreira et. al. ${ }^{30}$ avaliaram o perfil cognitivo em idosos institucionalizados por meio de três instrumentos, o Mini Exame do Estado Mental (MEEM), 8 o teste de trilhas $A$ que é composto por 25 círculos que possuem números de 1 a 25 , que devem ser colocados em sequência e seguindo a ordem. O idoso precisa ser ágil e fazer a tarefa o mais rápido que conseguir; o resultado 
é baseado no tempo levado para resolver cada parcela do teste. Para realização deste teste, é necessário uma velocidade adequada, planejamento motor e acuidade visual). ${ }^{31}$ O Clinical Dementia Rating (CDR) ou Escala de Demência, abrange a avaliação de 6 domínios e sua pontuação varia de 0 a 3 , sendo que zero considerado normal, 0,5 provável demência, 1 demência leve, 2 moderada e 3 grave. ${ }^{32}$

Durante a elaboração desta revisão sistemática, foram encontradas algumas limitações, como a escassez de informações recentes referente ao tema do artigo apresentado. Ressalta-se a importância de mais estudos nesta área, para auxílio aos profissionais e melhor entendimento do tema.

\section{CONSIDERACÕ̃ES FINAIS}

Conclui-se que todas as formas de avaliação aqui apresentadas mostraram-se de grande importância, pois são eficazes na detecção de deficiências e/ou incapacidades que podem estar presentes em idosos institucionalizados. A detecção correta de tais problemas se faz fundamental para um diagnóstico preciso e para a prescrição de condutas adequadas, direcionando a um tratamento satisfatório.

\section{REFERÊNCIAS}

1. Tissenbaum HA. Genetics, Life Span, Health Span, and the Aging Process in Caenorhabditis elegans. J Gerontol A Biol Sci Med Sci 2012;67A(5):503-510. doi: 10.1093/gerona/gls088

2. Zimmermann IMM, Leal MCC, Zimmermann RD, Marques APO, Gomes ECC. Fatores associados ao comprometimento cognitivo em idosos institucionalizados: revisão integrativa. Rev enferm UFPE 2015;9(12):1320-8. doi: 10.5205/ reuol.8127-71183-1-SM.0912201534

3. United Nations Department of Economic and Social Affairs/ Population Division 59 World Population Prospects: The 2015 Revision, Key Findings and Advance Tables. New York: UN; 2015.

4. Torres GV, Reis LA, Reis LA, Fernandes MH, Alves GS. Avaliação da capacidade de realização das atividades cotidianas em idosos residentes em domicílio. Revista Baiana 2009;33(3):466-475.

5. Gonçalves D, Altermann C, Vieira A, Machado AP, Fernandes R, Oliveira A, Mello-Carpes PB. Avaliação das funções cognitivas, qualidade de sono, tempo de reação e risco de quedas em idosos institucionalizados. Estudos Interdisciplinares sobre o envelhecimento 2014;19(1):95-108.

6. Nunciato AC, Pereira BC, Silva AB. Métodos de avaliação da capacidade física e qualidade de vida em idosos: revisão de literatura. Saúde em Revista, Piracicaba 2012;12(32):41-48, doi: $10.15600 / 22381244 /$ sr.v12n32p41-478

7. Reis LA, Rocha TS, Duarte SFP. Quedas: Risco e fatores associados em idosos. Revista Baiana de Enfermagem 2014;28(3):225-234.

8. Bourscheid FR, Mothes L, Irigaray TQ. Memória em idoso: relação entre percepção subjetiva e desempenho em testes objetivos. Estud. psicol. Campinas 2016;33(1).

9. Mcdowell I, Newell C. Measuring health: a guide to rating scales and questionnaires. 2nd ed. New York: Oxford University Press; 1996.
10. Podsiadlo D, Richardson S. The timed “Up \& Go": a test of basic functional mobility for frail elderly persons. J Am Geriatr Soc 1991;39(2):142-8. doi: 10.1111/j.1532-5415.1991.tb01616.x

11. Bretan O, Júnior JES, Ribeiro OR, Corrente JE. Risco de queda em idosos da comunidade: avaliação com o teste Timed up and go. Braz. j. otorhinolaryngol 2013;79(1):18-21. doi: 10.5935/1808-8694.20130004

12. Bacha JMR, Cordeiro LR, Alvisi TC, Bonfim TR. Impacto do treinamento sensório-motor com plataforma vibratória no equilíbrio e na mobilidade funcional de um indivíduo idoso com sequela de acidente vascular encefálico: relato de caso. Revista Fisioterapia e Pesquisa, São Paulo, Fisioter Pesq 2016;23(1):111-6. doi: 10.1590/1809 2950/14362423012016

13. Martins AS, Freire RC, Martins LH, Berton B, Júnior SAO, Christofolett G. Efeitos de uma terapia cognitivomotora em idosos institucionalizados. Rev Bras Ativ Fis e Saúde 2014;19(5):608-610. doi: 10.12820/rbafs.v.19n5p608

14. Rodrigues A.B, Yamashita E.T, Chiapetta A.L.M.L. Teste de fluência verbal no adulto e no idoso: Verificação da aprendizagem verbal. Revista CEFAC 2008;10(4):443-451.

15. Moura O, Simões MR, Pereira M. Fluência verbal semântica e fonêmica em crianças: funções cognitivas e análise temporal. Revista Avaliação Psicológica 2013;12(2):167-177.

16. Beato RG, Carvalho VA, Guimarães HC, Tumas V, Souza $\mathrm{CP}$, Oliveira GN, Caramelli P. Brazilian version of the Frontal Assessment Battery (FAB). Dementia e Neuropsychologia

17. 2007(1):59-65. doi 10.1590/S0004-282X2012005000009

18. Smanioto FN, Haddad MCFL. Índice de Katz aplicado a idosos institucionalizados. Rev Rene 2011;12(1):18-23.

19. Dias JÁ, Ovando AC, Külkamp W, Junior NGB. Força de preensão palmar: métodos de avaliação e fatores que influenciam a medida. Rev Bras Cineantropom Desempenho Hum 2010;12(3):209-216.

20. Tier CG, Santos SSC, Pelzer MT, Bulhosa MS. Escalas de avaliação da depressão em idosos. Revista Baiana de Enfermagem 2007;21(2/3):27-36.

21. Rosseto M, Maia KS, Silve VC, Pinto EC, Cosentino SF Soler MG. Depressão em idosos de uma instituição de longa permanência. Rev Enferm UFSM 2012;2(2):347-352. doi: $10.5902 / 217976924599$

22. Paiva ATG, Bessa MEP, Moraes GLA, Silva MJ, Oliveira RDP, Soares AMG. Avaliação da funcionalidade de famílias com idosos. Cogitare Enferm 2011;16(1):22-8. doi: 10.1590/ S0080-623420140000100008

23. Andreatini R, Seabra ML. A estabilidade do IDATE-traço: avaliação após cinco anos. Rev ABP-APAL 1993;15(1):21-5.

24. Corazza ST, Pereira ÉF; Dias JA. Creation, development and analysis of reproductiveness of test to evaluate simple and choice reaction times. The FIEP Bulletin 2007;77(1):613-615.

25. Camargos FFO, Dias RC, Dias JMD, Freire MTF. Adaptação transcultural e avaliação das propriedades psicométricas da Falls Efficacy Scale-International em idosos brasileiros (FES-I-BRASIL). Rev Bras Fisioter. Rev Bras Fisioter 2010;14(3):237-43.

26. Chellappa SL, Araújo JL. O sono e os transtornos do sono na depressão. Rev. Psiq. Clín 2007;34(6):285-289.

27. Cassis SVA, Karnakis T, Moraes TA, Curiati JAÉ, Quadrante ACR, Magaldi RM. Correlação entre o estresse do cuidados e as características clínicas do paciente portador de demência. Revisa da Associação Medicina Brasileira 2007;53(6):497501. doi: 10.1590/S0104-42302007000600015

28. Gomes MJ, Oliveira ERA, Paiva KM. Institucionalização 
e qualidade de vida de idosos da região metropolitana de Vitória/ES. Esc Anna Nery 2011;15(3):518-23. doi: 10.1590/ S1414- 81452011000300011

29. The Whoqol Group. Development of the World Health Organization WHOQOL-BREF Quality of Life Assessment. Psychological Medicine 1998;28:551-558.

30. Benedetti TRB, Mazo GZ, Gonçalves LHT. Bateria de testes da AAHPERD: adaptação para idosos institucionalizados. Rev Bras Cineantropom Desempenho Hum 2014,16(1):1-14. doi: 10.5007/1980-0037.2014v16n1p1
31. Ferreira LS, Pinho MSP, Pereira MWM, Ferreira AP. Perfil cognitivo de idosos residentes em Instituições de Longa Permanência de Brasília-DF. Rev Bras Enferm 2014;67(2):24751. doi:10.5935/0034-7167.20140033

32. Lezak MD (Org). Neuropsychological assessment. New York: Oxford University Press.1995.

33. Gil G, Busse AL. Avaliação neuropsicológica e o diagnóstico de demência, comprometimento cognitivo leve e queixa de memória relacionada à idade. Arq Med Hosp Fac Cienc Med Santa Casa São Paulo 2009;54(2):44-50. 